\title{
Effects of Time for Deliberation and Disfluency on Omission Neglect ${ }^{1}$
}

\author{
Ruomeng $\boldsymbol{W u}$ * \\ Assistant Professor \\ Department of Marketing \\ Gordon Ford College of Business, \\ Western Kentucky University \\ ruomeng.wu@wku.edu
}

\author{
Xiaoqi Han \\ Professor of Marketing, \\ Ancell School of Business, \\ Western Connecticut \\ State University
}

Meng Liu

Independent researcher

hanx@wcsu.edu

\section{Frank R. Kardes}

Donald E. Weston Professor of Marketing at the Lindner College of Business, University of Cincinnati

frank.kardes@uc.edu

${ }^{*}$ corresponding author

\section{INTRODUCTION}

The background in which a product description appears should be designed carefully when marketers design visual marketing signs. Prior literature has demonstrated the importance of research on fonts and other visual presentations in marketing communications as they have been shown to significantly impact consumers' information processing, judgments, and decisions (Pieters, Warlop, and Wedel 2002; Dennis et al. 2010; Sundar, Wu, Kardes 2019; Yoon et al. 2014; Dynel 2011; Huddleston et al. 2018; Kim and Lennon 2008; Sundar, Gonsales, and Schafer 2018).

Prior research suggests that understanding the fluency and disfluency effect is important to predicting what drives consumer judgment and decision making in marketing communications with textual / visual messages on signage, billboards, and other forms of outdoor advertising (Sundar et al. 2019; Wu, Shah, and Kardes 2020). Fluency, or ease of processing, usually leads to more favorable evaluative and affective judgments (Lee and Labroo 2004). As a result, marketers often adopt easy-to-read information to facilitate feelings of fluency. For example, clear and readable messages are often adopted to increase the visibility and comprehensibility of marketing communications, and are believed to be especially appealing for outdoor advertisements, which are viewed, on average, for 5 to 10 seconds (Taylor, Franke, and Bang 2006; Morones 2016). Nevertheless, the benefits of disfluency, or the experience of difficulty in processing information, should also be recognized (Schwarz 2004). For instance, difficult-to-read lettering has been shown to improve syllogistic reasoning and analytic processing, which in turn improve the quality of information processing (Alter et al. 2007; Song and Schwarz 2008).

\footnotetext{
${ }^{1}$ Funding for this study was provided by the AACSRE Emerging Fellowship grant.
}

\section{Abstract /}

The use of disfluency in marketing signage has more complex effects than what past research suggests. Time plays an important role in consumer information processing of signage presented disfluently. Three experimental studies suggest that the effects of disfluency on the awareness of missing information, purchase likelihood, and likelihood of future surprise depend on whether consumers have more or less time to process the information. When they have a limited amount of time, disfluency improves their awareness of missing information, leading to not only a lower likelihood of immediate purchase but also less surprise when important omissions are revealed later. Nevertheless, the effects are attenuated when consumers have a greater amount of time.

Keywords /

disfluency; time; omission neglect; signage marketing 
In the current research, we investigate the effects of disfluency, arising from displays of special lettering and low word-background contrast, on consumers' judgment and decision making. First, we attempt to confirm the major characteristic of disfluency: difficulty to read or process (Alter et al. 2007; Song and Schwarz 2008). Then, we examine how this characteristic can impact information processing and judgment through the theoretical framework of omission neglect, the lack of awareness of missing information (Sanbonmatsu et al. 2003). Prior research demonstrates that disfluency reduces omission neglect in general (Sundar et al. 2019). Extending the discovery of this prior research, we examine the role of processing time in moderating the disfluency effect, as processing time is a critical variable both theoretically and practically. The amount of time that consumers attend to marketing stimuli may differ across contexts, individuals, and products and now though, consumers often have little time evaluating marketing information (Hobbs 2016). For instance, as they quickly pass by a billboard or scroll through feeds on the phone, they usually spend a limited amount of time viewing or pondering over the information. But in some cases, consumers can spend even more time evaluating marketing information due to the decreased cost of information search (Smith, Bailey and Brynjolfsson 1999). Besides factors in the marketing environment, individual traits (e.g., need for cognitive closure) may impact how much time consumers allocate to a task before judgment is reached (Heaton and Kruglanski 1991). In summary, it appears important to examine the effects of processing time on consumer judgment and decision making in the domain of marketing communications.

\section{THE ROLE OF DISFLUENCY IN}

\section{OMISSION NEGLECT}

Fluency, or ease of processing, usually enhances evaluations because the degree of positive evaluation is attributed not only to product features but also to the conceptual or perceptual fluency that consumers experience (Lee and Labroo 2004). Conceptual fluency refers to the ease with which an idea or an association comes to mind whereas perceptual fluency describes the ease of identifying the characteristics of a stimulus (Tversky and Kahneman 1973; Jacoby and Dallas
1981). In this research we focus on the subsequent effects following changes in perceptual fluency. The instances of perceptual fluency enhancing marketing communications are numerous. For example, visibility in signage can break through clutter by improving the readability of information (Taylor et al. 2006). On the contrary, disfluency, or difficulty in processing, is often believed to move affective and evaluative judgments including liking, credibility, and persuasiveness to a negative end (see Novemsky et al. 2007). Indeed, prior research on disfluency focuses mainly on its negative effects (see Gill, Swann, and Silvera 1998; Novemsky et al. 2007; Roggeveen and Johar 2002; Schwarz 2004; Weisbuch and Mackie 2009). Nevertheless, understanding disfluency effects is also important because disfluency is a common experience; wear and tear on billboards and other outdoor signs caused by inclement weather, for example, are expected to increase disfluency (Visual 2016).

This research examines how consumers' experience of disfluency alters their sensitivity to missing information. Due to omission neglect, or the failure to detect missing information in marketing communications, consumers tend to overestimate the value of presented information and underestimate that of missing information (Sanbonmatsu et al. 2003; Silvera et al. 2005; Unkelbach, Fiedler, and Freytag 2007). As a result, they often form extreme judgments held with great confidence and make immediate purchase decisions that they later regret (Kardes et al. 2006; Sanbonmatsu et al. 2003). While it may seem tempting for marketers to foster omission neglect by providing only favorable information in their marketing communications, doing so may hurt a brand's image in the long run because of the higher likelihood of future regret (Sanbonmatsu et al.; Wu, Escoe, and Kardes 2017; Wu, Shah, and Kardes 2016). Therefore, it is important to understand how to debias omission neglect or to heighten consumers' awareness of missing information. Prior research demonstrates that disfluency increases analytic processing, encourages individuals to question their first impressions, and contributes to problem solving (Alter et al. 2007; Song and Schwarz 2008). Given that omission detection also requires effortful, analytic processing, disfluency may heighten consumers' sensitivity to 
relevant information missing from product description and help them form less extreme judgments and decisions (Sanbonmatsu, Kardes, and Sansone 1991). This proposition is supported by recent research on signage and information processing that shows how difficult-to-read fonts increase one's awareness of missing information and ability to detect missing information, leading to lower evaluation extremity (Sundar et al. 2019).

\section{THE MODERATING EFFECT OF PROCESSING TIME}

We attempt to extend our understanding of the disfluency effect on omission neglect by exploring the influence of processing time. While consumers often spend a very short amount of time on surrounding ads and signs, they may pay more attention and are willing to spend more time when there is a wide price dispersion or when they make important buying decisions (Hobbs 2016; Kumar, Lang, and Peng 2005). As a result, the role of processing time is highly relevant to understanding how consumers process marketing communications.

Time has been investigated as an important factor in the perceptual fluency literature. Reber and Schwarz (2001) found that the positive fluency effect is more pronounced when the processing time is short $(.3,1$, and 3 seconds) and it disappears over longer periods (10 seconds). They further explain that fluency most clearly improves stimulus identifications when processing time is short. Besides, if the effect of fluency is more pronounced with a shorter processing time, the positive evaluation should be attributed to the fluency experience itself, but not the intrinsic attractiveness of the object. In a similar vein, disfluency, which is like a cognitive glitch, may also be more noticeable and impactful under time pressure. When sufficient time is available, the effect may dissipate because consumers accommodate the disfluency.

The potential disfluency effect as a function of time has also been discussed in the need for cognitive closure literature, which suggests that as processing time decreases the need for cognitive closure is likely to increase, leading to a higher reliance on easier-to-process information and immediately avail- able judgmental cues (Heaton and Kruglanski 1991; Kruglanski, Webster, and Klem 1993; Roets et al. 2015; Webster and Kruglanski 1994). As presented (vs. missing) information is easier to use, when consumers have limited time, they should be less sensitive to missing information (Sundar et al. 2019). As a result, their evaluations should be more extreme, and they are more likely to make immediate purchases of products with favorable evaluations. Despite the initial favorable outcomes to marketers, consumers should be more susceptible to future surprise and presumably a higher likelihood of regret when important omissions are revealed later. Nevertheless, as discussed before, since disfluency increases analytic processing, when information is presented in a disfluent manner, consumers should be more sensitive to missing information despite the short amount of processing time (Song and Schwarz 2008). On the contrary, when consumers have a long time, which encourages them to take more judgmental cues into considerations before reaching a solution, their sensitivity to missing information should already be heightened, with or without disfluency (Kruglanski and Freund 1983). Hence, the abundance of time should lead to an attenuated effect of disfluency on omission neglect.

To summarize, we predict that the effect of disfluency on omission detection should become more pronounced under the condition in which processing time is short (vs. long). When processing time is long, the debiasing effect should be attenuated. Our hypotheses follow:

$\mathrm{H}_{1}$ : When processing time is short, disfluency reduces omission neglect, leading to lower immediate purchase intentions and less future surprise.

$\mathrm{H}_{2}$ : When processing time is long, the effect of disfluency on omission neglect is attenuated.

\section{OVERVIEW OF STUDIES}

The proposed conceptual model is presented in Figure 1 (below). Across three experimental studies, we manipulate disfluency via the uniqueness of fonts and the text-background color contrasts. Study $1 \mathrm{~A}$ and Study $1 \mathrm{~B}$ show the key interactive effect between 
time and disfluency on omission neglect. Study 2 confirms the effect captured in the first two studies and extends it from information processing to behavioral intentions. We predict that when consumers have a limited amount of time to view product information, disfluency will debias omission neglect by reducing perceived information sufficiency and increasing the likelihood to detect missing information, leading to lower immediate purchase intentions and less surprise if unexpected information is revealed later (Kardes et al. 2006; Sundar et al. 2019). Nevertheless, when a longer time is spent viewing product information, the difference between the disfluency and the fluency conditions will be attenuated or disappear.

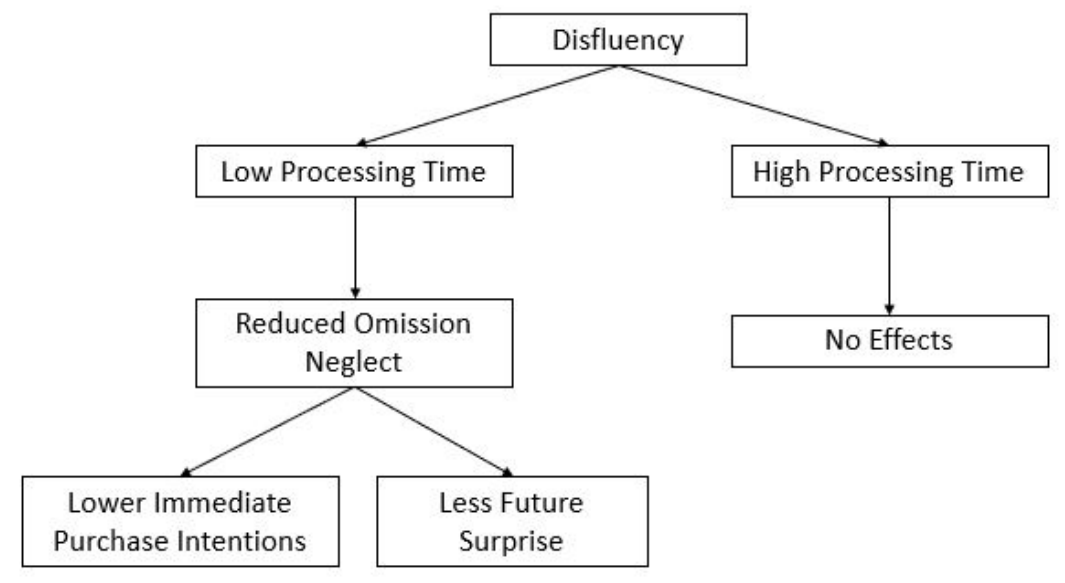

Figure 1 / The Conceptual Model

\section{STUDY 1A}

Study $1 \mathrm{~A}$ has a twofold purpose. First, we sought to replicate the debiasing effect of disfluency on omission neglect (Sundar et al. 2019). In particular, we predicted that information presented in a disfluent font would improve the awareness of missing information by decreasing the perceived sufficiency of the current information. Second, we tested our primary hypothesis concerning time's moderating effect. We predicted that when the information was easy to read, participants who spent a shorter time viewing the information would be less sensitive to missing information as they would concentrate on the presented information for easier judgmental cues. When the information was difficult to read, however, we predicted that participants would be more sensitive to missing information, even if they spent only a little time processing the available information. Moreover, as more time was spent processing the information, the differential impact between fluency and disfluency on omission detection / neglect should disappear.

\section{Method}

Participants consisted of 154 adults $\left(\mathrm{M}_{\text {age }}=35.23 ; 54.9 \%\right.$ female $)$ who were recruited via an online database (Amazon Mechanical Turk) and received a small amount of money to participate in the research. A 2 (font: difficult-to-read vs. easy-to-read) x 2 (time: shorter vs. longer) between-subject design was adopted. 
Participants were invited to evaluate a delivery app and based on a random assignment, participants had either a shorter or a longer time evaluating it. The time manipulation was adapted from Heaton and Kruglanski (1991). In the shorter-time condition, participants were informed that they would have only 5 seconds to view the information; it was explained that evaluating products must often be fast and a consumer often takes a very short time to evaluate a product. In the longer-time condition, participants were told that they could take as much time desired with the information. In this condition, it was stressed that evaluating products must be slow and that a careful consumer took a long time to evaluate a product. Participants were then randomly presented a billboard ad in either an easy- or difficult-to-read font (see Appendix A for the detailed stimuli). After viewing the information, all participants indicated how sufficient the information was for them to make an evaluation of the delivery app ( $1=$ not sufficient at all; $7=$ extremely sufficient). The higher the perceived sufficiency, the lower the awareness of missing information (Kardes et al. 2006; Sundar et al. 2019). They also reported how easy or difficult it was to read the information $(1=$ very easy; $7=$ very difficult $)$. Participants concluded by providing basic demographic information.

\section{Results}

\section{Manipulation checks}

A 2 x 2 (font $x$ time) analysis of variance performed on perceived difficulty revealed only a main effect of font $(F(1,150)=50.43, p<.001)$. Specifically, participants rated the information in the difficult-to-read condition as more difficult to read $(M=5.05, S D=$ $1.87)$ than that in the easy-to-read condition $(M=$ 2.97, $S D=1.79)$. Moreover, participants spent more time viewing the information in the longer-time condition $\left(M_{\text {shorter }}=4.86, S D=.77\right.$ vs. $M_{\text {longer }}=15.29, S D=$ $19.22 ; F(1,150)=22.59, p<.001)$.

\section{Sufficiency}

A $2 \times 2$ (font $\mathrm{x}$ time) analysis of variance performed on sufficiency yielded a main effect of font $(F(1,150)$ $=4.14, p=.044)$, such that participants perceived the information as more sufficient when it was easy to read $\left(M_{\text {easy }}=4.87, S D=1.57\right.$ vs. $\left.M_{\text {difficult }}=4.31, S D=1.83\right)$.
More importantly, there was a two-way interaction between font and time $(F(1,150)=4.87, p=.029$; see Figure 2). When the available time was shorter, perceived sufficiency was higher if the information was easier to read $\left(M_{\text {easy }}=5.13, S D=1.47\right.$ vs. $M_{\text {dificult }}$ $=3.97, S D=1.78 ; F=9.70, p=.003)$. When the time was longer, on the other hand, participants rated the information as equally sufficient, regardless of whether it was easy or difficult to read $\left(M_{\text {easy }}=4.59, S D=1.62\right.$ vs. $\left.M_{\text {difficult }}=4.64, S D=1.83 ; F<1\right)$.

\section{Sufficiency}

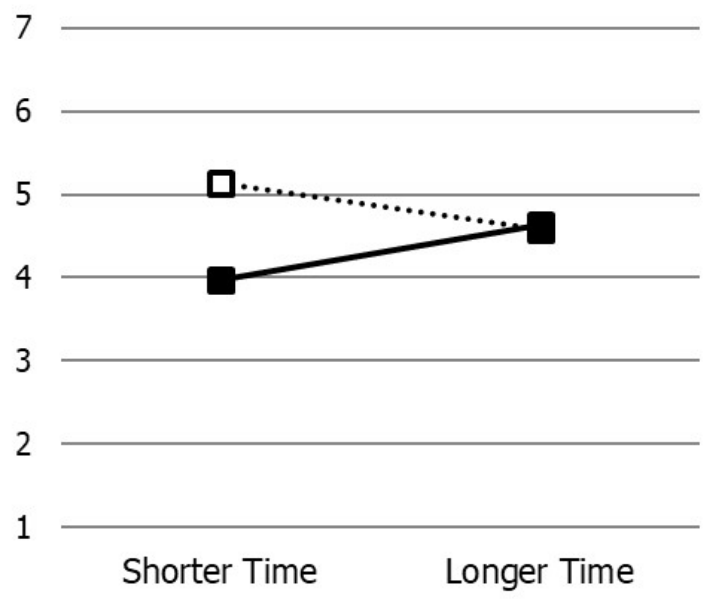

$\cdots \square \cdot$ Easy $\rightarrow$ - Diffculty

Figure 2 / Sufficiency as a Function of Font and Time in Study 1A

\section{Discussion}

In Study 1A, we investigated how disfluency interacts with time to impact omission neglect. We replicated Sundar et al's (2019) finding by showing that disfluency increases the awareness of missing information. More importantly, we found that the debiasing effect of disfluency is more pronounced when consumers do not have much time to evaluate the information and that the effect becomes attenuated when consumers spend a longer time processing the information carefully. Our findings are consistent with both the fluency and the need for cognitive closure literatures, such that when processing time is short (vs. long), disfluency stimulates consumers to consider a more complete set of judgmental cues before reaching a 
conclusion (Reber and Schwarz 2001; Kruglanski and Freund 1983; see Roets et al. 2015).

\section{STUDY 1B}

In Study 1B, we designed another scenario in which consumers were exposed to a billboard, and tested omission neglect via a more direct measure. Specifically, we asked participants to report how likely it was that relevant information was missing. Building upon the first study, we predicted that information presented in a disfluent word-background contrast would result in a heightened awareness of missing information when participants spent a limited amount of time viewing the information. When participants spent more time viewing the presented information, we expected that the effect of disfluency on omission neglect would become weaker.

In this study, we chose to measure processing time instead of manipulating it, thereby allowing idiosyncrasies, which contribute to variations in processing time, to come into play. Consumers can sometimes be forced to process information under time pressure as in Study 1A, but in other cases, they can freely decide how much time to attend to marketing stimuli. For instance, as they scroll through social media feeds, they may just skim over posts and spend little time digesting any single piece of information. When they make more serious decisions such as buying a car or viewing a job positing, however, their processing time may greatly increase. Even for the same product, processing time can differ across individuals based on their need for cognitive closure and other individual traits (see Heaton and Kruglanski 1991). Considering the practical relevance, we believe it is important to examine the effects of both manipulated and measured processing time.

\section{Method}

One-hundred three adult participants $\left(\mathrm{M}_{\text {age }}=35.47\right.$; $52.5 \%$ female) were recruited via an online database (Amazon Mechanical Turk) and received a small amount of money to participate in the research. A 2 (contrast: difficult-to-read vs. easy-to-read) x time (continuous) between-subject design was adopted. Participants were randomly assigned to one of the two contrast conditions.
Participants were invited to evaluate a protein bar based on a billboard advertisement. The procedure adopted to induce a contrast color-based disfluency was adapted from Sundar et al. (2019). Participants were randomly assigned to view the billboard information in an easy or difficult-to-read word-background contrast (see Appendix B for the detailed stimuli). Since participants were given as much processing time as desired, the processing time each participant spent on viewing the billboard ad was recorded. After viewing the ad, participants reported how likely relevant information was missing (1=extremely unlikely; $7=$ extremely likely) and how easy or difficult it was to read the information $(1=$ very easy; $7=$ very difficult). They concluded the study by reporting basic demographic information such as gender and age.

\section{Results}

\section{Manipulation check}

ANOVA performed on perceived difficulty revealed a main effect of contrast $(F(1,100)=74.10, p<.001)$. Specifically, participants rated the information in the difficult-to-read condition as more difficult to read $(M=5.88, S D=1.76)$ than that in the easy-to-read condition $(M=2.76, S D=1.89)$.

\section{Omission Detection}

We then submitted omission detection to a hierarchical regression analysis with time, contrast, and their interaction as predictors. Prior to the interaction analysis, we centered time by setting the mean to 0 . The main effect terms were entered in step 1, and the two-way interaction term was entered in step 2 . Coding was used for the contrast (easy-to-read $=0$, difficult-to-read $=1$ ). This procedure followed the recommendations from Cohen et al. (2003). The analyses revealed the key interactive effect between contrast and time $(B=-.13, t(99)=-2.45, p=.016$; see Figure 3 ). Using simple slope analysis, as demonstrated by Aiken and West (1991), we found that when the processing time was shorter $(0.7$ seconds, $1 S D$ below the mean), participants detected more missing information if the billboard was difficult to read $(B=1.00, t(99)=2.19, p=.031)$. When more time was spent viewing the information (13.52 seconds, 1 $S D$ above the mean), there was no difference between the two contrast conditions $(B=-.60, t(99)=-1.29$, 


\section{Omission Detection}

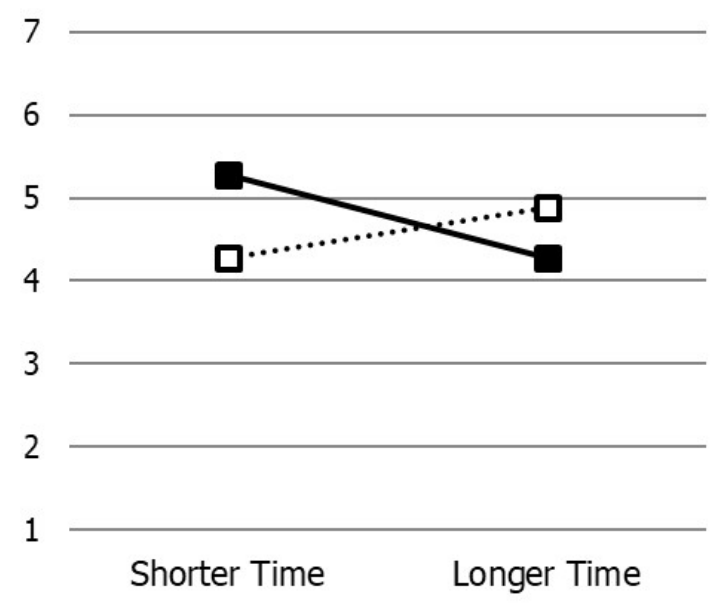

$\cdot \square \cdot$ Easy $\rightarrow$ Difficult

Figure 3 / Omission Detection as a Function of Font and Time in Study 1B

\section{Discussion}

Study $1 B$ confirms the debiasing effect of disfluency on omission neglect when processing time is short. Further, when information is presently fluently, consumers tend to be more sensitive to missing information if their processing time is longer. The pattern of the results shows no debiasing effect of disfluency when the time is greater ( $1 S D$ above the mean). In other words, when processing time becomes longer, consumers appear to be equally (in)sensitive to missing information regardless of disfluency or fluency.

Looking at the pattern of results in greater detail, we observed a backfiring effect of disfluency when consumers spend a very long time viewing the sign. Specifically, during an excessively long processing time (19.92 seconds; $2 S D$ above the mean), disfluency reduced omission detection $(B=-1.40, t(99)=-1.91$, $p=.059)$, suggesting that they showed even a greater tendency of omission neglect. The backfiring tendency potentially emerges because when information is presented disfluently and consumers with sufficient time attempt to interpret the information more effortfully. In other words, they may direct too much effort toward the disfluent presentation in order to see through it. This possibility is consistent with the self-regulation theory, which suggests that as difficulty increases, individuals allocate more cognitive effort to the focal task (Brehm and Self 1989; Nielsen and Escalas 2010). That effort may lead them to focus just on the presented information and neglect information not available at the time; processing disfluency has indeed been shown to result in more favorable (extreme) product evaluations, a consequence of heightened omission neglect (Nielsen and Escalas; Unkelbach et al. 2007). Based on the finding from this study and prior research, we suspect that when processing time is excessively long, disfluency may backfire by reducing sensitivity to missing information.

\section{STUDY 2}

After showing the interactive effect on omission neglect between disfluency and time, we sought to investigate the effect on behavioral intention. It was anticipated that when consumers spent a limited amount of time viewing the billboard, disfluent presentations, as compared to fluent ones, would heighten the awareness of missing information. This awareness would, in turn, reduce their immediate purchase intentions but also reduce the likelihood of future regret. Additionally, we tested perceived prettiness to rule out the possibility that participants had lower purchase intentions simply because they thought the ad was unattractive. We attempted to show that the effect on purchase intentions is due to heightened awareness of missing information, not the information's reduced attractiveness.

\section{Method}

One-hundred two participants $\left(M_{\text {age }}=35.96 ; 58 \%\right.$ female) were recruited via an online resource (Amazon Mechanical Turk). This study adopted a 2 (contrast: difficult- vs. easy-to-read) x processing time (continuous) between-subject design. Participants were randomly assigned to one of the two contrast conditions.

Participants were invited to evaluate a delivery app based on a billboard advertisement. They were randomly assigned to view information in an easy- 
or difficult-to-read word-background contrast (see Appendix $\mathrm{C}$ for the detailed stimuli) and we recorded the processing time each participant spent on viewing the billboard ad. After viewing the ad, participants reported their intentions to order deliveries through the advertised app $(1=$ not likely at all; $7=$ very likely $)$, how sufficient the information was for them to make an evaluation of the app (1= not sufficient at all; $7=$ extremely sufficient), how pretty the design was ( $1=$ very ugly; $7=$ very pretty). They also reported how easy or difficult it was to read ( $1=$ very easy; $7=$ very difficult $)$ and how much attention was given to the information on the billboard $(1=$ very little; $7=$ very $m u c h)$.

Next, on a separate page, we asked participants to imagine that they later found out this delivery app is rated 3 out of 5 by online reviewers. Following this, they reported how much they agreed or disagreed that this piece of information was unexpected (1=strongly disagree; $7=$ strongly agree $)$. As with the other studies, basic demographic information was collected.

\section{Results}

\section{Manipulation checks}

ANOVA performed on perceived difficulty revealed a main effect of contrast $(F(1,99)=112.57, p<.001)$. Specifically, participants rated the information in the difficult-to-read condition as more difficult to read ( $M$ $=5.00, S D=1.53)$ than the easy-to-read condition $(M$ $=2.02, S D=1.27)$. The disfluency manipulation did not impact the attention paid to the billboard ( $F(1$, $99)=1.83, p=.18)$. As expected, a regression analysis showed that the longer participants stayed on the page viewing the billboard, the more attention they paid to it in general $(B=.03, t(97)=2.13, p=.036)$.

\section{Purchase Intentions}

We then submitted purchase intentions to a hierarchical regression analysis with time, contrast, and their interaction as predictors. The analyses showed no main effects ( $p s>.24$ ) but revealed the proposed interactive effect between contrast and time $(B=.11, t(97)=2.11$, $p=.037$; see Figure 4$)$. Using the simple slope analysis from Aiken and West (1991), we found that when the time was shorter ( 4.33 seconds; $1 S D$ below the mean), participants showed lower purchase intentions when

\section{Purchase Intentions}

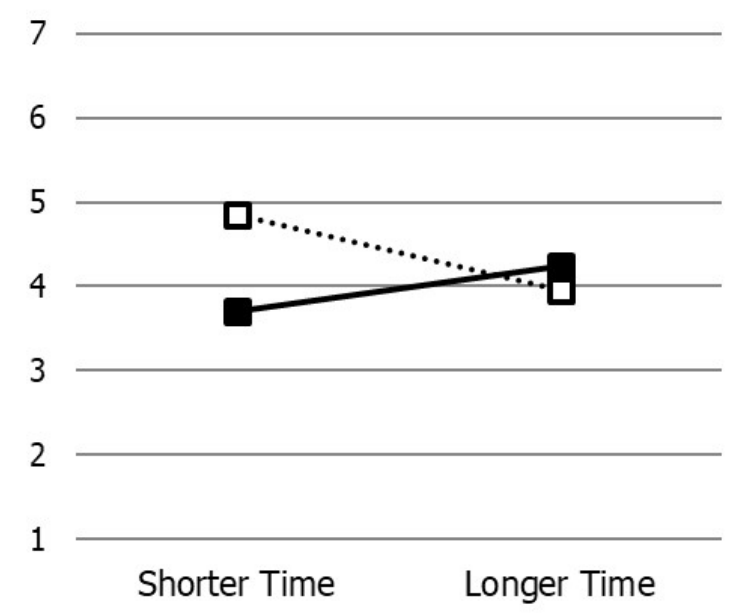

*ロ.. Easy $\rightarrow$ Difficult

Figure 4 / Purchase Intentions as a Function of Font and Time in Study 2

the billboard was difficult to read $(B=-1.14, t(97)$ $=-2.35, p=.021)$. When the time was longer $(17.82$ seconds; $1 S D$ above the mean), no difference was observed between the two contrast conditions ( $B=$ $.29, t(97)=.62, p=.54)$.

\section{Sufficiency}

The analyses on sufficiency showed no main effects ( $p s>.24)$ but revealed the proposed interactive effect between contrast and time $(B=.14, t(97)=2.73, p$ $=.008$; see Figure 5). Using simple slope analysis, we found that when the time was shorter ( 4.33 seconds; $1 S D$ below the mean), participants perceived the information as less sufficient if the billboard was difficult (vs. easy) to read $(B=-1.43, t(97)=-2.80, p$ $=.006)$. When the time was longer (17.82 seconds; $1 S D$ above the mean), no difference was observed between the two contrast conditions $(B=.50, t(97)=.1 .02, p$ $=.31$ ).

\section{Surprise}

Consistently, the analyses performed on perceived surprise showed no main effects $(p s>.75)$ but revealed the proposed interactive effect between contrast and time $(B=.10, t(97)=2.07, p=.041$; see Figure 6$)$. With a slope analysis, we found that when time was shorter 


\section{Sufficiency}

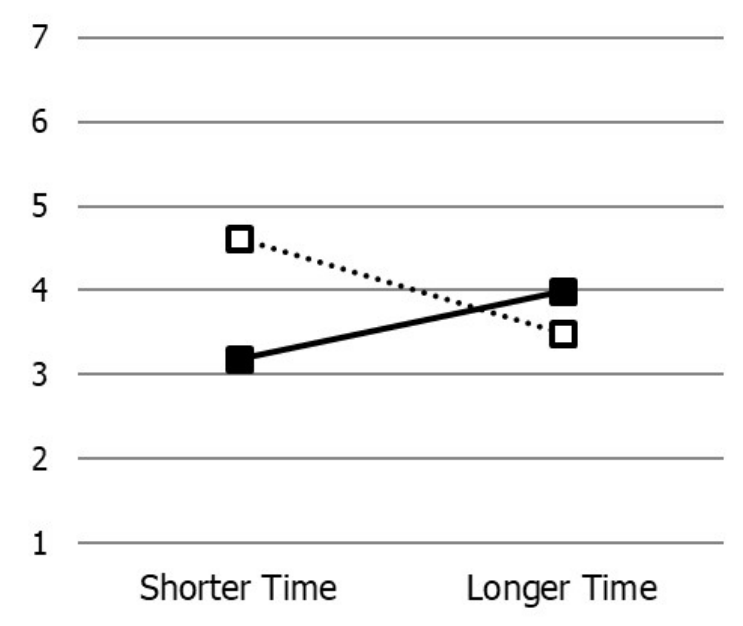

$\cdot \square \cdot$ Easy $\rightarrow-$ Difficult

Figure 5 / Sufficiency as a Function of Font and Time in Study 2

(4.33 seconds; $1 S D$ below the mean), participants experienced less surprise afterward when the billboard was difficult (vs. easy) to read $(B=-1.01, t(97)=-1.86$, $p=.066)$. When the time was longer (17.82 seconds;

\section{Surprise}

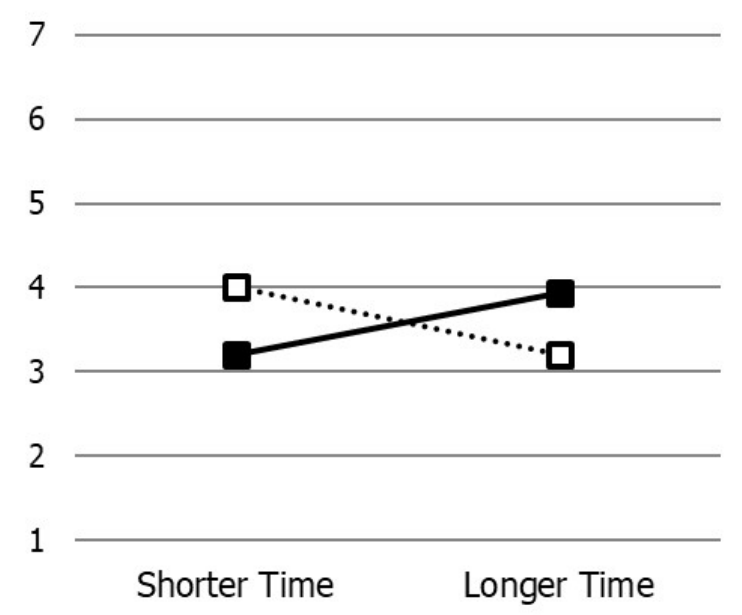

\section{$\cdot \square \cdot$ Easy $\rightarrow$ Difficult}

Figure 6 / Surprise as a Function of Font and Time in Study 2
$1 S D$ above the mean), no effect of the contrast was observed $(B=.54, t(97)=1.22, p=.23)$.

\section{Mediation analyses}

Next, we used bootstrapping procedures to assess the extent to which the interactive effect on purchase intentions and surprise are driven by omission neglect (Hayes 2018). The results revealed significant mediating pathways for both variables (indirect effect $_{\text {purchase intentions }}=.09,95 \%$ CI: .0211, .1481; indirect effect $_{\text {surprise }}=.04,95 \%$ CI: .0055, .0851). In other words, sufficiency, as a measure of omission neglect (Kardes et al. 2006), increased immediate purchase likelihood but resulted in surprise later.

\section{Prettiness}

The analyses on perceived prettiness showed no interactive effect between contrast and time $(B=.08$, $t(97)=1.48, p=.14)$. This result ruled out prettiness as an alternative explanation.

\section{DISCUSSION}

Study 2 replicates the proposed interactive effect between disfluency and processing time, yet in another context. Disfluency reduces omission neglect by decreasing perceived information sufficiency when consumers spend only a limited amount of time evaluating the ad. This decreased omission neglect reduces the immediate purchase likelihood, but also the likelihood of surprise when the missing information is revealed later. When consumers spend a longer time viewing the stimuli, the difference between disfluency and fluency disappears. The pattern of the results suggests that the debiasing effect of disfluency become more effective among individuals who choose to process information faster. In this study, we have also ruled out perceived prettiness as an alternative explanation for the effects.

Additionally, as shown through the pattern in Study 1B, disfluency shows a backfiring tendency by increasing the susceptibility to omission neglect when processing time becomes much more abundant. Specifically, when the time became very long (20.22 seconds; $2 S D$ above the mean), disfluency led to marginally higher perceived sufficiency $(B=1.47, t(97)=1.86, p=.066)$ and more surprise $(B=1.21, t(97)=1.71, p=.09)$. 


\section{GENERAL DISCUSSION}

This research investigates how processing time impacts the way consumers interpret disfluent information. It reconciles the effects of disfluency on deliberative, analytic processing through omission neglect (see Alter et al. 2007; Unkelbach et al. 2007). Past research suggests that disfluency can increase one's awareness of missing information, leading to improved information processing and decision making (Sundar et al. 2019). Our research extends the omission neglect literature by identifying the role of processing time, as both a manipulated, dichotomous variable and a measured, continuous variable (see Unkelbach et al.). As variations exist in the amount of time individuals need to encode and comprehend information before judgment or a decision is made, studying processing time as a continuous variable offers a higher degree of external validity. We find that with limited processing time, disfluency results in decreased omission neglect and improved decisions at the time the judgment or choice is made. Under this circumstance, consumers have lower immediate purchase intentions and experience less unfavorable surprise once exposed to omissions. When they spend more time, however, the difference between the disfluency and the fluency conditions is attenuated, and when processing time becomes overly long, disfluency even backfires by increasing omission neglect; disfluency may require consumers to direct more effort to the presented information at the expense of noticing missing attributes. This interesting pattern potentially contributes to backfiring research (Sanna and Schwarz 2003; 2006; Sanna, Schwarz, and Stocker 2002).

Besides the theoretical contributions, our research is of practical importance to both businesses and consumers. Presenting information fluently through easy-to-read designs is often perceived as beneficial for printed ads or billboards because difficulty can lead to lowered evaluations (Gill et al. 1998). This may seem especially true for billboards, which are viewed for but a few seconds, or when consumers do not have much time viewing the information (Morones 2016). However, our research suggests that in order to facilitate more prudent information processing, marketers should strategically take advantage of conceptual or perceptual disfluency, allowing consumers to notice missing information and form less extreme, albeit more stable, evaluations of a brand. For example, when the quality of a consumer's decision (e.g., a medical choice or a refund request) matters to both the company and the clients, marketers can use disfluency to trigger a less extreme product evaluation and a more cautious purchase decision.

Our research consistently suggests that although easy-to-read signage can induce consumers to focus more on the presented favorable information, such omission neglect may lead to more surprise later and that surprise may negatively impact customer satisfaction and repurchase likelihood in the long term. The benefits of using difficult-to-process designs may become especially relevant when marketers want to initiate and maintain long-term relationships with consumers (see Alter et al. 2007). For example, mature brands that value long-term relationships over first impressions may find 
disfluent presentations in outdoor signs or other visual marketing communications more beneficial. When the evaluation time of a sign is limited, disfluency created through special fonts or low word-background contrast colors can encourage consumers to consider a more complete set of criteria before making a conclusion, therefore improving the quality of their decisions.

While marketers may assume that more time improves information processing and decision quality, the current research suggests this is not always so. When consumers spend too much time evaluating a sign, disfluent presentations may backfire by shifting attention to the presented information. Therefore, when consumers make important decisions and are known to take a long time evaluating a set of criteria carefully, it may be effective to present information in an easy-to-process way as disfluency may increase their susceptibility to omission neglect. Similarly, consumers can benefit from our research by understanding how they may be impacted by the time they spend on marketing stimuli and the information's format.

This research focused on advertising billboards, for which consumers may not have complete control over how much time they have to view and process information. Future research might explore how time and disfluency impact information processing and decision making in other marketing contexts (e.g., marketing through smartphones, laptops, or tablets) where consumers have more control over the pace of information flow. The practical importance of our research might be extended to digital technologies where consumers quickly scroll through information. We would expect factors such as individual traits to have a bigger impact in a context where consumers have more control over the pace of information processing. Prior research, for example shows that individuals with a higher level of conscientiousness more readily adjust to greater task difficulty by allocating more effort to the task (Yeo and Neal 2008). Based on our findings, they may be less receptive to the debiasing effect of disfluency. Since processing time and the need for cognitive closure are closely related but remain different constructs, it is worth exploring how the need for cognitive closure might interact with disfluency to impact decisions through omission neglect (Kruglanski and Freund 1983).

Finally, our research indicates an interesting backfiring effect of disfluency on omission neglect when processing time becomes overly long. While we focus on the debiasing role of disfluency when the processing time is short in the current article, the backfiring effect might be worth exploring in greater detail in future research, which could look at more theoretical and practical implications of disfluency's effects across different time spans to guide future marketing designs. Through this work we offer a new, theoretical account of how consumers respond to disfluent presentations of information as a function of processing time. Our investigation presents new opportunities for future theoretical research and we encourage more practical research to study the parameters regarding the topic. We hope our contributions to the field of signage will inspire future research to continue the advancements suggested in this article. 


\section{REFERENCES}

Alter, A. L., Oppenheimer, D. M., Epley, N., and Eyre, R. N. (2007). Overcoming intuition: metacognitive difficulty activates analytic reasoning. Journal of Experimental Psychology: General 136, 569-576.

Brehm, J. W. and Self, E. A. (1989). The intensity of motivation. Annual Review of Psychology 40(1), 109-131.

Cohen, J., Cohen, P., West, S. G., and Aiken, L. S. (eds). (2013). Applied Multiple Regression/Correlation Analysis for the Behavioral Sciences. Mahwah: Lawrence Erlbaum.

Dennis, C., Newman, A., Michon, R., Brakus, J. J., and Wright, L. T. (2010). The mediating effects of perception and emotion: Digital signage in mall atmospherics. Journal of Retailing and Consumer Services 17, 205-215.

Dynel, M. (2011). Blending the incongruity-resolution model and the conceptual integration theory: The case of blends in pictorial advertising. International Review of Pragmatics 3, 59-83.

Gill, M. J., Swann Jr, W. B., and Silvera, D. H. (1998). On the genesis of confidence. Journal of Personality and Social Psychology 75(5), 1101-1114.

Hayes, A. F. (2018). Introduction to Mediation, Moderation, and Conditional Process Analysis: A Regression-Based Approach. New York: Guilford.

Heaton, A. W. and Kruglanski, A. W. (1991). Person perception by introverts and extraverts under time pressure: Effects of need for closure. Personality and Social Psychology Bulletin 17, 161-165.

Hobbs, T. (2016). "Marketers continue to 'waste money' as only 9\% of digital ads are viewed for more than a second." Marketing Week, 26 June. https://www.marketingweek.com/marketers-continue-to-waste-money-as-only-9-of-digital-ads-are-viewed-for-morethan-a-second/

Huddleston, P. T., Behe, B. K., Driesener, C., and Minahan, S. (2018). Inside-outside: Using eye-tracking to investigate search-choice processes in the retail environment. Journal of Retailing and Consumer Services 43, 85-93.

Jacoby, L. L. and Dallas, M. (1981). On the relationship between autobiographical memory and perceptual learning. Journal of Experimental Psychology: General 110(3), 306.

Kardes, F. R., Posavac, S. S., Silvera, D., Cronley, M. L., Sanbonmatsu, D. M., Schertzer, S., and Chandrashekaran, M. (2006). Debiasing omission neglect. Journal of Business Research 59, 786-792.

Kim, M. and Lennon, S. (2008). The effects of visual and verbal information on attitudes and purchase intentions in internet shopping. Psychology and Marketing 25, 146-178.

Kumar, N., Lang, K. R., and Peng, Q. (2005). Consumer search behavior in online shopping environments. Proceedings of the 38th Annual Hawaii International Conference on System Sciences 3(3), 87-102.

Kruglanski, A. W. and Freund, T. (1983). The freezing and unfreezing of lay-inferences: Effects on impressional primacy, ethnic stereotyping, and numerical anchoring. Journal of Experimental Social Psychology 19, 448-468.

Kruglanski, A. W., Webster, D. M., and Klem, A. (1993). Motivated resistance and openness to persuasion in the presence or absence of prior information. Journal of Personality and Social Psychology 65, 861-876.

Lee, A. Y., and Labroo, A. A. (2004). The effect of conceptual and perceptual fluency on brand evaluation. Journal of Marketing Research $41,151-165$.

Morones, H. (2008). 5 rules of effective billboard design and advertising. 99 Designs. https://99designs.com/blog/tips/billboard-design-tips/

Nielsen, J. H., and Escalas, J. E. (2010). Easier is not always better: The moderating role of processing type on preference fluency. Journal of Consumer Psychology 20, 295-305.

Novemsky, N., Dhar, R., Schwarz, N., and Simonson, I. (2007). Preference fluency in choice. Journal of Marketing Research 44, 347-356.

Pieters, R., Warlop, L., and Wedel, M. (2002). Breaking through the clutter: Benefits of advertisement originality and familiarity for brand attention and memory. Management Science 48, 765-781.

Reber, R. and Schwarz, N. (2001). The hot fringes of consciousness: Perceptual fluency and affect. Consciousness and Emotion 2, $223-231$.

Roets, A., Kruglanski, A. W., Kossowska, M., Pierro, A., and Hong, Y. Y. (2015). The motivated gatekeeper of our minds: New directions in need for cognitive closure theory and research. Advances in Experimental Social Psychology 52, 221-283.

Roggeveen, A. L., and Johar, G. V. (2002). Perceived source variability versus familiarity: Testing competing explanations for the truth effect. Journal of Consumer Psychology 12, 81-91. 
Sanbonmatsu, D. M., Kardes, F. R., Houghton, D. C., Ho, E. A., and Posavac, S. S. (2003). Overestimating the importance of the given information in multiattribute consumer judgment. Journal of Consumer Psychology 13(3), 289-300.

Sanbonmatsu, D. M., Kardes, F. R., and Sansone, C. (1991). Remembering less and inferring more: Effects of time of judgment on inferences about unknown attributes. Journal of Personality and Social Psychology 61(4), 546-554.

Sanna, L. J. and Schwarz, N. (2003). Debiasing the hindsight bias: The role of accessibility experiences and (mis) attributions. Journal of Experimental Social Psychology 39, 287-295.

Sanna, L. J., and Schwarz, N. (2006). Metacognitive experiences and human judgment: The case of hindsight bias and its debiasing. Current Directions in Psychological Science 15, 172-176.

Sanna, L. J., Schwarz, N., and Stocker, S. L. (2002). When debiasing backfires: Accessible content and accessibility experiences in debiasing hindsight. Journal of Experimental Psychology: Learning, Memory, and Cognition 28, 497-302.

Schwarz, N. (2004). Metacognitive experiences in consumer judgment and decision making. Journal of Consumer Psychology 14(4), $332-348$.

Silvera, D. H., Kardes, F. R., Harvey, N., Cronley, M. L., and Houghton, D. C. (2005). Contextual influences on omission neglect in the fault tree paradigm. Journal of Consumer Psychology 15(2), 117-126.

Smith, M. D., Bailey, J., and Brynjolfsson, E. (1999). Understanding digital markets: review and assessment. MIT Press, Cambridge.

Song, H. and Schwarz, N. (2008). Fluency and the detection of misleading questions: Low processing fluency attenuates the Moses illusion. Social Cognition 26, 791-799.

Sundar, A., Wu, R., and Kardes, F. R. (2019). Faded fonts. Interdisciplinary Journal of Signage and Wayfinding 3, $16-27$.

Sundar, A., Gonsales, F., and Schafer, G. (2018). Synchronicity in signage promotes a sense of belonging. Interdisciplinary Journal of Signage and Wayfinding 2(2), 30-40.

Taylor, C. R., Franke, G. R., and Bang, H. K. (2006). Use and effectiveness of billboards: perspectives from selective-perception theory and retail-gravity models. Journal of Advertising 35, 21-34.

Tversky, A. and Kahneman, D. (1973). Availability: A heuristic for judging frequency and probability. Cognitive Psychology 5(2), $207-232$.

Unkelbach, C., Fiedler, K., and Freytag, P. (2007). Information repetition in evaluative judgments: Easy to monitor, hard to control. Organizational Behavior and Human Decision Processes 103(1), 37-52.

Visual, P. (2016). Common signage problems and how to deal with them. PB Visual, 27 April. http://pbvisual.com.au/how-to-deal-with4-common-signage-problems/

Webster, D. M. and Kruglanski, A. W. (1994). Individual differences in need for cognitive closure. Journal of Personality and Social Psychology, 67, 1049-1062.

Weisbuch, M. and Mackie, D. (2009). False fame, perceptual clarity, or persuasion? Flexible fluency attribution in spokesperson familiarity effects. Journal of Consumer Psychology 19, 62-72.

$\mathrm{Wu}, \mathrm{R} ., \mathrm{Escoe}, \mathrm{B}$., and Kardes, F. (2017). You are not as smart as you think you are: Effects of self-perceived knowledge on consumer information processing and decision making. ACR North American Advances.

Wu, R., Shah, E., and Kardes, F. (2016). Disfluency effects on inference and evaluation. ACR North American Advances.

$\mathrm{Wu}, \mathrm{R}$., Shah, E., and Kardes, F. (2020). “The struggle isn't real:” How need for cognitive closure moderates inferences from disfluency. Journal of Business Research 109, 585-594.

Yeo, G. and Neal, A. (2008). Subjective cognitive effort: A model of states, traits, and time. Journal of Applied Psychology 93(3), 617-631.

Yoon, A. Choi, S.M., Hong, J., Hahn, D., Kang, M., and Lee, S. (2014). Motivational Signage Promotes Physical Activity on a College Campus. Medicine and Science in Sports and Exercise 46, 108. 


\section{APPENDICES}

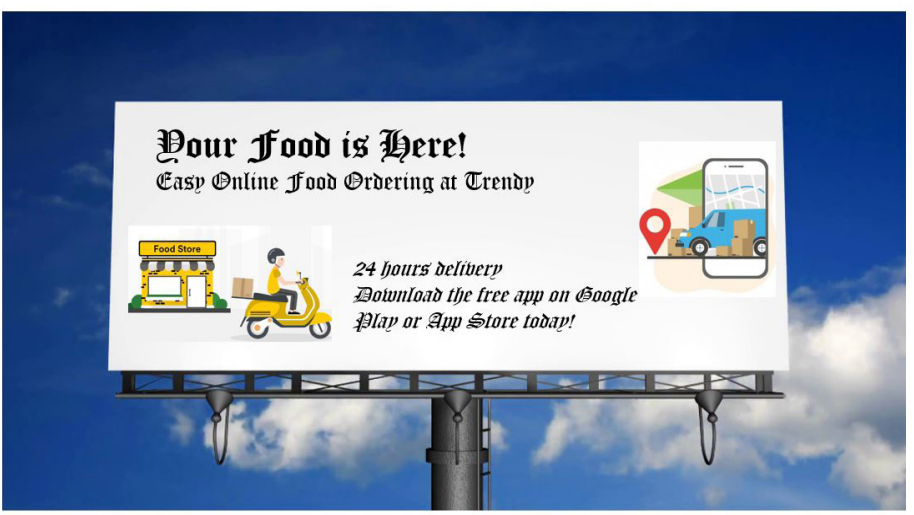

Appendix A / Difficult to Read Font

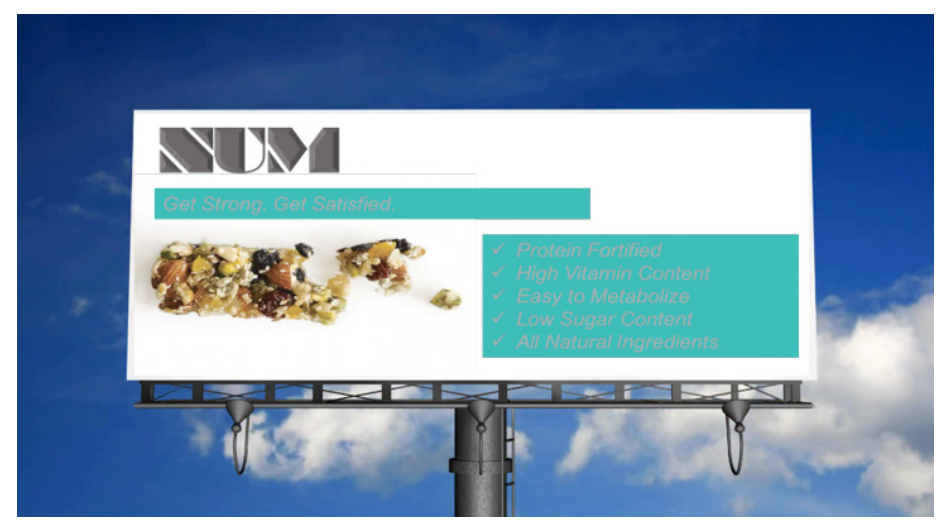

Appendix B / Difficult to Read Contrast

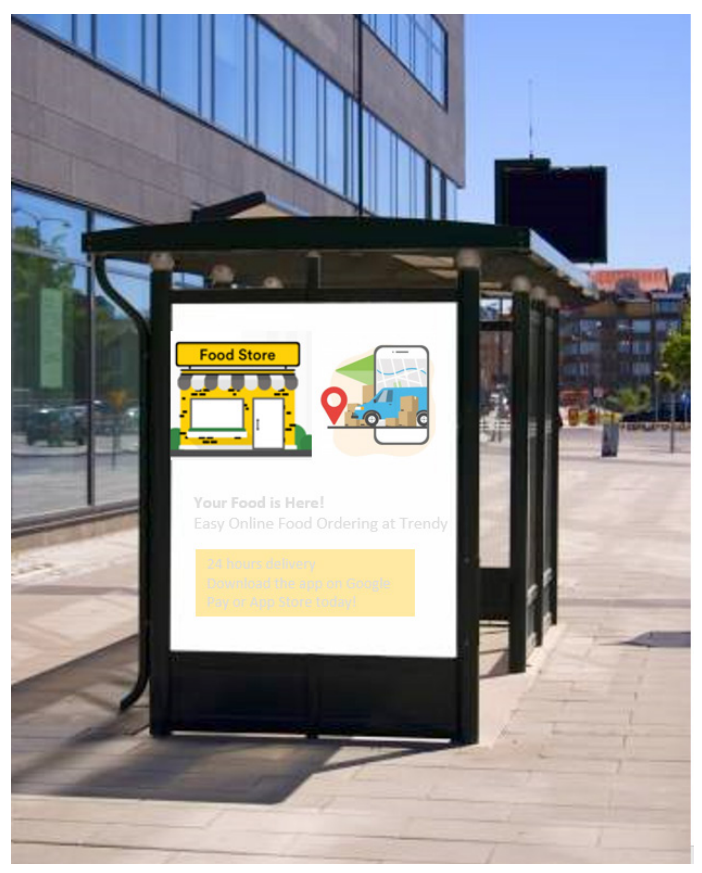

Appendix C / Difficulty to Read Contrast

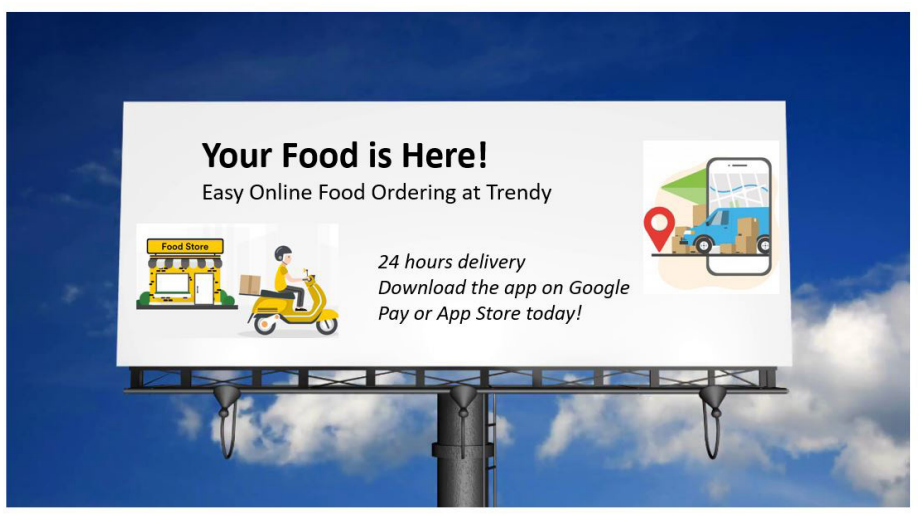

Appendix A / Easy to Read Font

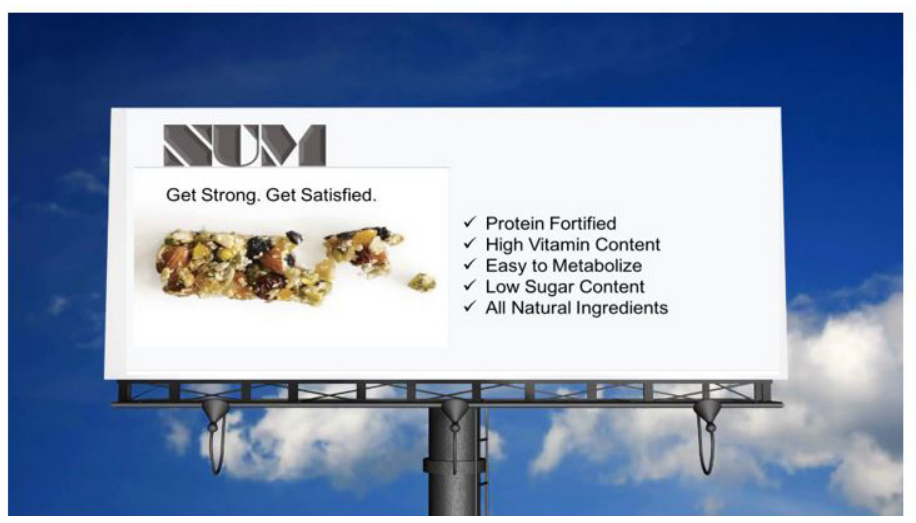

Appendix B / Easy to Read Contrast

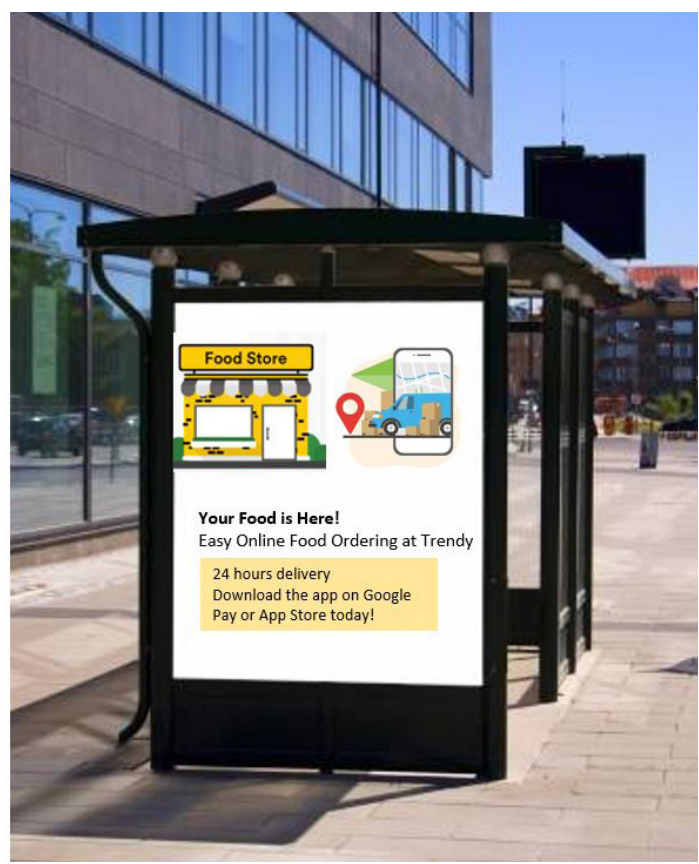

Appendix C / Easy to Read Contrast 